hep-th/0203277

KEK-TH-804

SAGA-HE-186

March 2002

\title{
Orbifold Matrix Model
}

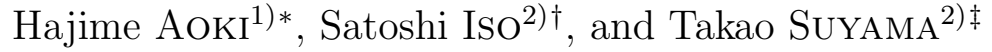 \\ 1) Department of Physics, Saga University, Saga 840-8502, Japan \\ 2) High Energy Accelerator Research Organisation (KEK), \\ Tsukuba, Ibaraki 305-0801, Japan
}

\begin{abstract}
We study a matrix model describing type IIB superstring in orbifold backgrounds. We particularly consider a $\mathbf{C}^{3} / \mathbf{Z}_{3}$ orbifold model whose six dimensional transverse space is orbifolded by $\mathbf{Z}_{3}$ discrete symmetry. This model is chiral and has $d=4 \mathcal{N}=1$ supersymmetry of Yang-Mills type as well as an inhomogeneous supersymmetry specific to matrix models. We calculate one-loop effective action around some backgrounds, and the result can be interpreted as interactions mediated by massless particles in IIB supergravity in orbifold background, if the background is in the Higgs branch. If the background is in the Coulomb branch, the dynamics is governed by the reduced model of $d=4$ super Yang-Mills theory, which might be interpreted as exchange of massless particles in the twisted sector. But the perturbative calculation does not reproduce the supergravity result. We also show that this model with a large Higgs vacuum expectation value becomes IIB (IKKT) matrix model.
\end{abstract}

\footnotetext{
* e-mail address : haoki@cc.saga-u.ac.jp

$\dagger$ e-mail address : satoshi.iso@kek.jp

$\ddagger$ e-mail address : tsuyama@post.kek.jp
} 


\section{Introduction}

A large $N$ reduced model has been proposed as a nonperturbative formulation of type IIB superstring theory [1]. It is defined by the action円:

$$
S=-\frac{1}{g^{2}} \operatorname{Tr}\left(\frac{1}{4}\left[A_{I}, A_{J}\right]\left[A^{I}, A^{J}\right]+\frac{1}{2} \bar{\psi} \Gamma^{I}\left[A_{I}, \psi\right]\right) .
$$

It is a large $N$ reduced model [2] of ten dimensional super Yang-Mills theory. Here $\psi$ is a ten dimensional Majorana-Weyl spinor field, and $A_{I}$ and $\psi$ are $N \times N$ Hermitian matrices. It is formulated in a manifestly covariant way which enables us to study the nonperturbative issues of superstring theory. In fact we can, in principle, predict the dimensionality of spacetime, the gauge group and the matter contents by solving this model [3].

Among several issues on the matrix formulation of superstring, one is to answer which model should be regarded as the most fundamental one. This question can be compared to the universality in the lattice gauge theory. In this case, the most important symmetry we have to keep is the gauge symmetry and we know that various models with the gauge invariance are in the same universality class. In the case of matrix models, maximal supersymmetry may play the same role but it is not yet certain. Hence, it is meaningful to consider various matrix models, investigate their dynamics and make connections between them. If matrix models can describe the dynamics of space-time, we should be able to generate various space-time backgrounds from the same model, and in this respect universality in matrix models will be related to the background independence.

Another related issue is to construct a phenomenologically interesting model with chiral fermions in $d=4$. In string theory, there are several mechanisms to generate fourdimensional chiral fermions, including compactification with non-zero index of Dirac operator, D-branes wrapped on intersecting cycles, etc [4]. In matrix models, we may similarly be able to consider corresponding mechanisms but no investigations have been performed yet.

In this paper we study a matrix model in $\mathbf{C}^{3} / \mathbf{Z}_{3}$ orbifold background [5] [6]. This model can be obtained from IIB matrix model by imposing some constrains. In particular we consider a model with four-dimensional $\mathcal{N}=1$ Yang-Mills type supersymmetry. In matrix models, there is also an inhomogeneous supersymmetry as well, and the model has totally $d=4 \mathcal{N}=2$ supersymmetries. This model is chiral since the chiral multiplets are in bifundamental representation of the gauge group. There are two types of classical solutions,

\footnotetext{
${ }^{1}$ In this paper, we take a metric convention $\eta_{I J}=\operatorname{diag}(-1,1, \cdots, 1)$.
} 
corresponding to the Higgs branch and the Coulomb branch, respectively. The Higgs branch solution forms a triplet under $\mathbf{Z}_{3}$ transformation and there are three images in $\mathbf{C}^{3}$ plane whose positions in $d=4$ directions are the same. On the other hand, the Coulomb branch solution is restricted on the orbifold fixed point and cannot move into $\mathbf{C}^{3}$ direction. The Coulomb branch solution is continuously connected to the Higgs branch solution at the orbifold singularity. When the Higgs branch solution comes on the orbifold singularity, the three images are liberated from each other and they can move independently into $d=4$ directions. As BPS solutions, the former represents ordinary D-branes with three mirror images by $\mathbf{Z}_{3}$, and when they lie on the singularity they can move freely into the four dimensional direction as fractional branes.

We calculate one-loop effective action of the orbifold matrix model to clarify its correspondence to the interaction in type IIB supergravity in the orbifold background [7]. Firstly we show that we can reproduce the correct supergravity interaction for classical solutions in the Higgs branch in orbifold background. Secondly the dynamics of fractional branes in the Coulomb branch is shown to be governed by the reduced model of $d=4$ super Yang-Mills theory instead of $d=10$ Yang-Mills theory. In string theory, fractional branes [8] have a charge of RR fields in the twisted sector and the interaction between them should have four-dimensional behaviour in the long distance. In this respect, it is qualitatively consistent with the string picture. But perturbatively the reduced model of $d=4$ super Yang-Mills theory does not reproduce the correct interactions mediated by massless fields in the twisted sector. (The induced interaction is inversely proportional to the fourth power of the distance instead of the expected quadratic power.) This discrepancy will be a limitation of perturbative calculations and it is not certain whether incorporation of nonperturbative effects can help it.

This model has smaller supersymmetries compared with IIB matrix model and it is not apparent if it is in the same universality class as IIB matrix model. But if it is, the orbifold matrix model must be obtained from IIB matrix model through condensation of some fields and so must be the reverse. These relations will also shed light on the issue how to realize four-dimensional chiral fermions from IIB matrix model. In this paper, we show that the orbifold matrix model in the Higgs phase with a large expectation value becomes IIB matrix model.

The organisation of the paper is as follows. In section 2, we give a brief review of IIB matrix model. In section 3, we construct orbifold matrix models, and particularly 
consider a model with $\mathcal{N}=1$ supersymmetry of Yang-Mills type in $d=4$. In section 4 , we analyse this orbifold matrix model. First we list several classical solutions with or without noncommutativity of backgrounds. Then we calculate one-loop effective action around these solutions and show that the result is partly consistent with the string theory. In section 5, we show that this model with a large Higgs vacuum expectation value becomes IIB matrix model. Section 6 is devoted to conclusions and discussions.

\section{Brief Review of IIB Matrix Model}

Action of the IIB matrix model is defined in (1.1). This model has $\mathcal{N}=2$ ten-dimensional supersymmetry if we regard the distribution of eigenvalues as space-time. Since this matrix model action can be obtained by reducing the original ten dimensional space-time to a single point, the action has the remnant of the supersymmetry of super Yang-Mills type:

$$
\begin{aligned}
\delta^{(1)} \psi & =\frac{i}{2}\left[A_{I}, A_{J}\right] \Gamma^{I J} \epsilon, \\
\delta^{(1)} A_{I} & =i \bar{\epsilon} \Gamma^{I} \psi .
\end{aligned}
$$

In addition to this homogeneous symmetry, there is an inhomogeneous fermionic symmetry

$$
\begin{aligned}
\delta^{(2)} \psi & =\xi \\
\delta^{(2)} A_{I} & =0 .
\end{aligned}
$$

If we take a linear combination of $\delta^{(1)}$ and $\delta^{(2)}$ as

$$
\begin{aligned}
& \tilde{\delta}^{(1)}=\delta^{(1)}+\delta^{(2)}, \\
& \tilde{\delta}^{(2)}=i\left(\delta^{(1)}-\delta^{(2)}\right),
\end{aligned}
$$

we obtain the $\mathcal{N}=2$ supersymmetry algebra:

$$
\begin{aligned}
\left(\tilde{\delta}_{\epsilon}^{(i)} \tilde{\delta}_{\xi}^{(j)}-\tilde{\delta}_{\xi}^{(j)} \tilde{\delta}_{\epsilon}^{(i)}\right) \psi & =0 \\
\left(\tilde{\delta}_{\epsilon}^{(i)} \tilde{\delta}_{\xi}^{(j)}-\tilde{\delta}_{\xi}^{(j)} \tilde{\delta}_{\epsilon}^{(i)}\right) A_{I} & =2 i \bar{\epsilon} \Gamma^{I} \xi \delta_{i j}
\end{aligned}
$$

The $\mathcal{N}=2$ supersymmetry is a crucial element of superstring theory. It imposes strong constraints on the spectra of particles. Furthermore it determines the structure of the interactions uniquely in the light-cone string field theory [9]. The IIB matrix model is a nonperturbative formulation which possesses such a symmetry. Therefore it has a very good chance 
to capture the universality class of IIB superstring theory. These symmetry considerations force us to interpret the eigenvalues of $A_{I}$ as the space-time coordinates.

The simplest classical solutions are those where all bosonic matrices are commutable and simultaneously diagonalisable. Since the distributions of the eigenvalues determine the extent and the dimensionality of spacetime, the structure of spacetime can be dynamically determined by the theory; Indeed, spacetime exits as a single bunch and no single eigenvalue can escape from the rest [10]. Numerical simulations and analysis with various approximations have indicated that the Lorentz symmetry might be spontaneously broken [10, 11].

The next simplest solutions are those with non-zero but c-number commutator $\left[A_{I}, A_{J}\right]=$ $i \theta_{I J} \mathbf{1}_{N \times N}$. These solutions correspond to BPS-saturated backgrounds. Indeed, the solutions are invariant under transformations if we set $\xi$ equal to $\pm \frac{1}{2} \theta_{I J} \Gamma^{I J} \epsilon$ in the $\mathcal{N}=2$ supersymmetry (2.1) and (2.2). By expanding the matrix around this classical solution, we can obtain a supersymmetric noncommutative gauge theory [12].

Finally in this section, we comment on the calculation of the one-loop effective action in IIB matrix model and its interpretation in type IIB supergravity. In [四], it was shown that when the bosonic matrices are expanded around backgrounds having a block-diagonal form

$$
A_{\mu}=X_{\mu}=\left(\begin{array}{ll}
d_{\mu(1)}+X_{\mu(1)} & \\
& d_{\mu(2)}+X_{\mu(2)}
\end{array}\right),
$$

the one-loop effective action becomes

$$
\begin{aligned}
W^{(12)}= & \frac{1}{4\left(d^{(1)}-d^{(2)}\right)^{8}} \\
& \left(\left[\left(-4 n_{2} \operatorname{Tr}\left(\tilde{f}_{\mu \nu}^{(1)} \tilde{f}_{\nu \lambda}^{(1)} \tilde{f}_{\lambda \rho}^{(1)} \tilde{f}_{\rho \mu}^{(1)}\right)-8 n_{2} \operatorname{Tr}\left(\tilde{f}_{\mu \nu}^{(1)} \tilde{f}_{\lambda \rho}^{(1)} \tilde{f}_{\mu \rho}^{(1)} \tilde{f}_{\lambda \nu}^{(1)}\right)\right.\right.\right. \\
& +2 n_{2} \operatorname{Tr}\left(\tilde{f}_{\mu \nu}^{(1)} \tilde{f}_{\mu \nu}^{(1)} \tilde{f}_{\lambda \rho}^{(1)} \tilde{f}_{\lambda \rho}^{(1)}\right)+n_{2} \operatorname{Tr}\left(\tilde{f}_{\mu \nu}^{(1)} \tilde{f}_{\lambda \rho}^{(1)} \tilde{f}_{\mu \nu}^{(1)} \tilde{f}_{\lambda \rho}^{(1)}\right) \\
& \left.-48 \operatorname{Tr}\left(\tilde{f}_{\mu \nu}^{(1)}\right) \operatorname{Tr}\left(\tilde{f}_{\mu \lambda}^{(2)} \tilde{f}_{\lambda \rho}^{(2)} \tilde{f}_{\rho \mu}^{(2)}\right)+12 \operatorname{Tr}\left(\tilde{f}_{\mu \nu}^{(1)}\right) \operatorname{Tr}\left(\tilde{f}_{\mu \nu}^{(2)} \tilde{f}_{\lambda \rho}^{(2)} \tilde{f}_{\lambda \rho}^{(2)}\right)+(1 \leftrightarrow 2)\right] \\
& \left.-48 \operatorname{Tr}\left(\tilde{f}_{\mu \nu}^{(1)} \tilde{f}_{\nu \lambda}^{(1)}\right) \operatorname{Tr}\left(\tilde{f}_{\mu \rho}^{(2)} \tilde{f}_{\rho \lambda}^{(2)}\right)+6 \operatorname{Tr}\left(\tilde{f}_{\mu \nu}^{(1)} \tilde{f}_{\mu \nu}^{(1)}\right) \operatorname{Tr}\left(\tilde{f}_{\lambda \rho}^{(2)} \tilde{f}_{\lambda \rho}^{(2)}\right)\right) \\
& +O\left(\left(1 /\left(d^{(1)}-d^{(2)}\right)^{9}\right)\right.
\end{aligned}
$$

where $f_{\mu \nu}^{(i)}=i\left[X_{\mu}^{(i)}, X_{\nu}^{(i)}\right]$ and we keep terms which vanish for finite $N$. (These terms play an important role in calculating interactions between objects with charges of antisymmetric fields such as D-branes.) Observing the tensor structures, we find the exchanges of massless particles in IIB supergravity corresponding to graviton, scalars and antisymmetric fields. In this paper, we perform similar calculations in the matrix model in orbifold backgrounds. 


\section{$3 \quad \mathrm{C}^{3} / \mathrm{Z}_{3}$ Orbifold Matrix Model}

\section{1 $\quad \mathrm{Z}_{3}$ Orbifolding}

We now construct matrix models in $\mathbf{C}^{3} / \mathbf{Z}_{3}$ orbifold background [6]. The IIB matrix model consists of ten hermitian bosonic matrices $A_{I}(I=0, \cdots, 9)$ and $d=10$ Majorana-Weyl fermionic matrices with sixteen components. We take complex coordinates for the sixdimensional transverse space and write them as $B_{i}(i=1,2,3)$,

$$
B_{1}=A_{4}+i A_{5}, \quad B_{2}=A_{6}+i A_{7}, \quad B_{3}=A_{8}+i A_{9} .
$$

We characterise the action of $\mathbf{Z}_{3}$ group by three integers $\left(a_{1}, a_{2}, a_{3}\right)$. The $\mathbf{Z}_{3}$ transformation is defined to act on these complex coordinates as $B_{i} \rightarrow \omega^{a_{i}} B_{i}$. Different choices of these integers lead to different models with a different number of unbroken supersymmetries in $d=4$.

From the D-instanton point of view, since there are three images of each D-instanton, we have to extend the size of the matrices three times to represent these three mirror images. The $\mathbf{Z}_{3}$ group rotates these three images. We write hermitian matrices as tensor products of $3 \times 3$ matrices on which $\mathbf{Z}_{3}$ acts and the rest. The $3 \times 3$ matrices can be expanded in terms of 't Hooft matrices,

$$
U=\left(\begin{array}{ccc}
1 & & \\
& \omega & \\
& & \omega^{2}
\end{array}\right), \quad V=\left(\begin{array}{lll}
0 & 0 & 1 \\
1 & 0 & 0 \\
0 & 1 & 0
\end{array}\right),
$$

where $U V=\omega V U$. In a representation where the coordinates of each mirror image of $\mathrm{D}$ instantons is diagonalized, the $\mathbf{Z}_{3}$ transformation interchanges these diagonal blocks, and is expressed as $M \rightarrow V M V^{\dagger}$, where $M$ is $A_{I}$ or $\psi$. While this representation is geometrically clear, for later convenience, we take another representation where the $\mathbf{Z}_{3}$ transformation is given as $M \rightarrow U M U^{\dagger}$.

We now impose $\mathbf{Z}_{3}$ invariance conditions on matrices. The $\mathbf{Z}_{3}$ condition for the gauge field $\left(A_{\mu}, \mu=0,,, 3\right)$ is

$$
A_{\mu}=U A_{\mu} U^{\dagger}
$$

The off-diagonal blocks are projected out and only the diagonal blocks $A_{\mu(i, i)}, i=1,2,3$, of $3 \times 3$ matrices survive after this $\mathbf{Z}_{3}$ projection. If the size of each block matrix is $N \times N$, these gauge fields are associated with $U(N)^{3}$ symmetry. The $\mathbf{Z}_{3}$ invariance condition for the complex fields $B_{i}$ is given by

$$
B_{i}=\omega^{a_{i}} U B_{i} U^{\dagger}
$$


and is written as $a_{i}+i-j=0(\bmod 3)$ for $(i, j)$ blocks.

Fermionic matrices are similarly projected out by this $\mathbf{Z}_{3}$ transformation. A convenient way to pick up $\mathbf{Z}_{3}$ invariant components out of the original $d=10$ Majorana-Weyl fermionic matrix $\psi$ is to write it in lower dimensional Weyl-basis. Since $d=10$ chirality operator $\Gamma=\Gamma^{01 \cdots 9}$ is written as a product of lower dimensional chirality operators as $\Gamma=\left(i \Gamma^{0123}\right)\left(i \Gamma^{45}\right)\left(i \Gamma^{67}\right)\left(i \Gamma^{89}\right)$, a positive chirality fermion $\psi$ in $d=10$ can be decomposed as a sum of the fermions $\psi=\sum_{i=0}^{3}\left(\psi_{L, i}+\psi_{R, i}\right)$ in the following table.

\begin{tabular}{|c||c|c|c|c||c|c|c|}
\hline$\Gamma$ & $i \Gamma^{0123}$ & $i \Gamma^{45}$ & $i \Gamma^{67}$ & $i \Gamma^{89}$ & & Majorana & $b_{i}$ \\
\hline \hline \multirow{6}{*}{+} & + & + & + & + & $\psi_{L, 0}$ & $\psi_{0}$ & $\left(a_{1}+a_{2}+a_{3}\right) / 2$ \\
\cline { 2 - 7 } & + & + & - & - & $\psi_{L, 1}$ & $\psi_{1}$ & $\left(a_{1}-a_{2}-a_{3}\right) / 2$ \\
\cline { 2 - 7 } & + & - & + & - & $\psi_{L, 2}$ & $\psi_{2}$ & $\left(-a_{1}+a_{2}-a_{3}\right) / 2$ \\
\cline { 2 - 7 } & + & - & - & + & $\psi_{L, 3}$ & $\psi_{3}$ & $\left(-a_{1}-a_{2}+a_{3}\right) / 2$ \\
\cline { 2 - 7 } & - & - & - & - & $\psi_{R, 0}$ & $\left(\psi_{0}\right)^{c}$ & $\left(-a_{1}-a_{2}-a_{3}\right) / 2$ \\
\cline { 2 - 7 } & - & - & + & + & $\psi_{R, 1}$ & $\left(\psi_{1}\right)^{c}$ & $\left(-a_{1}+a_{2}+a_{3}\right) / 2$ \\
\cline { 2 - 7 } & - & + & - & + & $\psi_{R, 2}$ & $\left(\psi_{2}\right)^{c}$ & $\left(a_{1}-a_{2}+a_{3}\right) / 2$ \\
\cline { 2 - 7 } & - & + & + & - & $\psi_{R, 3}$ & $\left(\psi_{3}\right)^{c}$ & $\left(a_{1}+a_{2}-a_{3}\right) / 2$ \\
\hline
\end{tabular}

Since the $d=10$ charge conjugation interchanges left and right as $\left(\psi_{L, i}\right)^{c}=\left(\psi^{c}\right)_{R, i}$, a $d=10$ Majorana-Weyl fermion $\psi$ contains four independent complex degrees of freedom $\psi_{L, i}$, and the right-handed fermions are related to the left as $\psi_{R, i}=\left(\psi_{L, i}\right)^{c}$. In the following we write $\psi_{L, i}$ without index $L$, and $\psi_{R, i}$ as its charge conjugation. Hence the fermionic $3 N \times 3 N$ matrix $\psi$ is decomposed as

$$
\psi=\sum_{i=0}^{3}\left(\psi_{i}^{A}+\left(\psi_{i}^{A}\right)^{c}\right) T^{A}
$$

where $T^{A}$ 's are generators of $3 N \times 3 N$ hermitian matrices. Since the $\mathbf{Z}_{3}$ transformation acts on each complex plane $\mathbf{C}$ in $\mathbf{C}^{3}, \mathbf{Z}_{3}$ invariant condition depends on how each fermion transforms under rotations in each $\mathbf{C}$ plane. Hence, according to the eigenvalues of $i \Gamma^{45}, i \Gamma^{67}$ and $i \Gamma^{89}$, each of the fermionic matrices is subject to the following $\mathbf{Z}_{3}$ invariance condition:

$$
\psi_{i}=\omega^{b_{i}} U \psi_{i} U^{\dagger}
$$

where the charges $b_{i}$ 's are calculated from $a_{i}$ 's as (3.5). Invariant blocks of $3 \times 3$ matrices satisfy $b_{i}+i-j=0(\bmod 3)$.

An action of the orbifold matrix model is given from the IIB matrix model action (1.1) by restricting the matrices by the $Z_{3}$ constraints (3.3), (3.4) and (3.7). First we rewrite the IIB matrix model action (1.1) in terms of the $d=4$ gauge field and the complex coordinate 
fields. The bosonic part of IIB matrix model action becomes

$$
S_{b}=-\frac{1}{4 g^{2}} \operatorname{Tr}\left(\left[A_{\mu}, A_{\nu}\right]^{2}+2 \sum_{i=1}^{3}\left[A_{\mu}, B_{i}\right]\left[A^{\mu}, B_{i}^{\dagger}\right]+\frac{1}{2} \sum_{i, j=1}^{3}\left(\left[B_{i}, B_{j}^{\dagger}\right]\left[B_{i}^{\dagger}, B_{j}\right]+\left[B_{i}, B_{j}\right]\left[B_{i}^{\dagger}, B_{j}^{\dagger}\right]\right)\right) .
$$

The $\operatorname{Tr}$ is a trace over $3 N \times 3 N$ matrices. The bosonic part of the orbifold matrix model action is given by (3.8) where matrices are constrained by the conditions (3.3), (3.4). The potential term for $B_{i}$ field can be written as

$$
-\frac{1}{2} \operatorname{Tr}\left(\left|\sum_{i}\left[B_{i}, B_{i}^{\dagger}\right]\right|^{2}+2 \sum_{i, j}\left|\left[B_{i}, B_{j}\right]\right|^{2}\right)
$$

and each term in this expression comes from the integration of $\mathrm{D}$ term and $\mathrm{F}$ term respectively in the superspace formalism. Comparing to the $N \times N$ IIB matrix model (1.1), the $3 N \times 3 N$ orbifold matrix model has three times as many as matrices.

The fermionic part of the IIB matrix model action (1.1) is rewritten in terms of the four dimensional fermions in the table 3.5$)$ as

$$
S_{f}=-\frac{1}{2 g^{2}} \operatorname{Tr}\left(\sum_{i=0}^{3} \overline{\psi_{i}} \Gamma^{\mu}\left[A_{\mu}, \psi_{i}\right]+2 \sum_{i=1}^{3} \overline{\left(\psi_{i}\right)^{c}} \bar{\Gamma}^{(i)}\left[B_{i}^{\dagger}, \psi_{0}\right]+\sum_{i, j, k=1}^{3}\left|\epsilon_{i j k}\right| \overline{\left(\psi_{i}\right)^{c}} \Gamma^{(j)}\left[B_{j}, \psi_{k}\right]+\text { h.c. }\right)
$$

where we have defined complex gamma matrices

$$
\Gamma^{(1)} \equiv \frac{1}{2}\left(\Gamma^{4}-i \Gamma^{5}\right), \quad \bar{\Gamma}^{(1)} \equiv \frac{1}{2}\left(\Gamma^{4}+i \Gamma^{5}\right),
$$

and similarly for $\Gamma^{(2)}$ and $\Gamma^{(3)}$. The fermionic part of the orbifold matrix model is given by (3.10) where fermionic matrices are restricted by the condition (3.7).

By $\mathbf{Z}_{3}$ orbifolding, the original symmetry of IIB matrix model with size $3 N \times 3 N$ is generally reduced from $S O(9,1) \times U(3 N)$ to $S O(3,1) \times U(N)^{3}$. $\mathbf{Z}_{3}$ invariant fermion fields transform as bi-fundamental representations under the unbroken $U(N)^{3}$ gauge symmetry and the resulting gauge theory becomes a so-called quiver gauge theory. A subgroup of the transverse $S O(6)$ symmetry is also unbroken. It depends on the charge assignment $a_{i}$. A model in the next subsection has unbroken $U(3)$ symmetry, which rotate three chiral superfields.

As for supersymmetry, we decompose the supersymmetry parameter $\epsilon$ and $\xi$ of (2.1) and (2.2) into the lower dimensional Weyl basis in the table (3.5). If some of these supersymmetry parameters are invariant under the $\mathbf{Z}_{3}$ transformation, they transform $\mathbf{Z}_{3}$ invariant fields among themselves. Therefore, the number of zero charges of $b_{i}(i=0, \cdots, 3)$ is the 
number of unbroken four dimensional supersymmetries. While in matrix models, we have inhomogeneous supersymmetries (2.2) as well as the Yang-Mills type (2.1), in this paper, we will name the model by the number of Yang-Mills type supersymmetry.

\section{2 $\mathcal{N}=1$ Model}

We particularly consider a model with $\mathbf{Z}_{3}$ charge assignment $a_{i}=2$ for $i=1,2$, 3 , and accordingly $b_{0}=0, b_{i}=2(i=1,2,3)$. The $\mathbf{Z}_{3}$ invariance conditions (3.3) (3.4)(3.7) can be satisfied if we restrict the matrices to the following form

$$
\begin{aligned}
& A_{\mu}=\left(\begin{array}{ccc}
A_{\mu}^{(1)} & 0 & 0 \\
0 & A_{\mu}^{(2)} & 0 \\
0 & 0 & A_{\mu}^{(3)}
\end{array}\right), \quad B_{i}=\left(\begin{array}{ccc}
0 & 0 & B_{i}^{(2)} \\
B_{i}^{(3)} & 0 & 0 \\
0 & B_{i}^{(1)} & 0
\end{array}\right), \quad B_{i}^{\dagger}=\left(\begin{array}{ccc}
0 & B_{i}^{(3) \dagger} & 0 \\
0 & 0 & B_{i}^{(1) \dagger} \\
B_{i}^{(2) \dagger} & 0 & 0
\end{array}\right), \\
& \psi_{0}=\left(\begin{array}{ccc}
\psi_{0}^{(1)} & 0 & 0 \\
0 & \psi_{0}^{(2)} & 0 \\
0 & 0 & \psi_{0}^{(3)}
\end{array}\right), \psi_{i}=\left(\begin{array}{ccc}
0 & 0 & \psi_{i}^{(2)} \\
\psi_{i}^{(3)} & 0 & 0 \\
0 & \psi_{i}^{(1)} & 0
\end{array}\right), \\
& \left(\psi_{0}\right)^{c}=\left(\begin{array}{ccc}
\left(\psi_{0}\right)^{c(1)} & 0 & 0 \\
0 & \left(\psi_{0}\right)^{c(2)} & 0 \\
0 & 0 & \left(\psi_{0}\right)^{c(3)}
\end{array}\right), \quad\left(\psi_{i}\right)^{c}=\left(\begin{array}{ccc}
0 & \left(\psi_{i}\right)^{c(3)} & 0 \\
0 & 0 & \left(\psi_{i}\right)^{c(1)} \\
\left(\psi_{i}\right)^{c(2)} & 0 & 0
\end{array}\right) .
\end{aligned}
$$

Inserting these expansions into (3.8) and (3.10), we can obtain the explicit form of the action for the orbifold matrix model.

As we will see at the end of this subsection, this model has $\mathcal{N}=1$ Yang-Mills type supersymmetry. $A_{\mu}$ and $\psi_{0}$ fields form a vector multiplet while $B_{i}$ and $\psi_{i}$ fields make three chiral multiplets. The left and right handed fermions in the chiral multiplets $\left(\psi_{i}\right.$ and $\left.\left(\psi_{i}\right)^{c}\right)$ are in different bifundamental representations of $U(N)^{3}$ and hence the model is chiral in $d=4$ and phenomenologically attractive. It is also interesting to consider other models with different charge assignments, symmetries, and chirality.

We can also write the $\mathbf{Z}_{3}$ invariant fields as

$$
\begin{aligned}
& A_{\mu}=\sum_{a=0,1,2} A_{\mu}^{a} \otimes U^{a}, \quad B_{i}=\sum_{a=0,1,2} B_{i}^{a} \otimes\left(U^{a} V\right), \\
& \psi_{0}=\sum_{a=0,1,2} \psi_{0}^{a} \otimes U^{a}, \quad \psi_{i}=\sum_{a=0,1,2} \psi_{i}^{a} \otimes\left(U^{a} V\right),
\end{aligned}
$$

where $U$ and $V$ are the 't Hooft matrices (3.2). Since $A_{\mu}$ 's are hermitian, $\left(A_{\mu}^{a}\right)^{\dagger}=A_{\mu}^{-a}$. Charge conjugated and hermitian conjugated fields are expanded as

$$
B_{i}^{\dagger}=\sum_{a=0,1,2} B_{i}^{a \dagger} \otimes\left(V^{\dagger} U^{-a}\right)
$$




$$
\begin{aligned}
& \left(\psi_{0}\right)^{c}=\sum_{a=0,1,2}\left(\psi_{0}^{a}\right)^{c} \otimes U^{-a}, \quad\left(\psi_{i}\right)^{c}=\sum_{a=0,1,2}\left(\psi_{i}^{a}\right)^{c} \otimes\left(V^{\dagger} U^{-a}\right), \\
& \overline{\psi_{i}}=\sum \overline{\psi_{i}^{a}} \otimes\left(V^{\dagger} U^{-a}\right), \quad \overline{\left(\psi_{i}\right)^{c}}=\sum_{a=0,1,2} \overline{\left(\psi_{i}^{a}\right)^{c}} \otimes\left(U^{a} V\right) .
\end{aligned}
$$

These expansions are more useful than the block representation (3.12) when we investigate this model in the next section.

Since only $b_{0}$ vanishes, there is one $d=4$ Yang-Mills type supersymmetry in this model. Inhomogeneous fermionic transformations (2.2) also make the orbifold matrix model action invariant only if the supersymmetry parameter $\xi$ is the $\psi_{0}$ type. The Yang-Mills type supersymmetry of this orbifold matrix model is given by

$$
\begin{aligned}
\delta^{(1)} \psi_{0} & =\frac{i}{2}\left(\left[A_{\mu}, A_{\nu}\right] \Gamma^{\mu \nu}+\left[B_{i}, B_{i}^{\dagger}\right]\right) \epsilon_{0}, \\
\delta^{(1)} \psi_{i} & =\frac{i}{2}\left(\left|\epsilon_{i j k}\right|\left[B_{j}^{\dagger}, B_{k}^{\dagger}\right] \bar{\Gamma}^{(j)} \bar{\Gamma}^{(k)} \epsilon_{0}+2\left[A_{\mu}, B_{i}\right] \Gamma^{\mu} \Gamma^{(i)}\left(\epsilon_{0}\right)^{c}\right) \\
\delta^{(1)} A_{\mu} & =i \overline{\epsilon_{0}} \Gamma^{\mu} \psi_{0}+i \overline{\left(\epsilon_{0}\right)^{c}} \Gamma^{\mu}\left(\psi_{0}\right)^{c} \\
\delta^{(1)} B_{i} & =2 i \overline{\left(\epsilon_{0}\right)^{c}} \bar{\Gamma}^{(i)} \psi_{i} .
\end{aligned}
$$

The other supersymmetry is

$$
\delta^{(2)} \psi_{0}=\xi_{0}
$$

Here $\epsilon_{0}$ and $\xi_{0}$ are supersymmetry parameters restricted to the $\psi_{0}$ type component in the table (3.5). Combining them similarly to (2.3), they become $d=4 \mathcal{N}=2$ space-time supersymmetry whose commutator gives translation of $A_{\mu}(\mu=0,, 3)$. In matrix models, bosonic fields are interpreted as space-time coordinates and hence the above supersymmetry can be identified with the $\mathcal{N}=2$ four-dimensional space-time supersymmetry. In this paper, however, we call this model $\mathcal{N}=1$ orbifold matrix model, counting only the number of Yang-Mills type supersymmetries.

\section{Investigations of $\mathcal{N}=1$ Orbifold Matrix Model}

\subsection{Classical Solutions}

We now investigate the orbifold matrix model, especially $\mathcal{N}=1$ type model. Equations of motion can be obtained from the equations of motion of IIB matrix model $\left[A_{I},\left[A_{I}, A_{J}\right]\right]=0$ by restricting $A_{I}$ to the $\mathbf{Z}_{3}$ invariant parts. This is because the projected-out fields by $\mathbf{Z}_{3}$ symmetry always enter in the action with another projected-out field. 
We first search for commutable solutions $\left[A_{I}, A_{J}\right]=0$. This equation can be written in terms of the $\mathbf{Z}_{3}$ invariant components in the block representation (3.12) as

$$
\begin{aligned}
& {\left[A_{\mu}^{(\alpha)}, A_{\nu}^{(\alpha)}\right]=0,} \\
& A_{\nu}^{(1)} B_{i}^{(2)}-B_{i}^{(2)} A_{\nu}^{(3)}=A_{\nu}^{(2)} B_{i}^{(3)}-B_{i}^{(3)} A_{\nu}^{(1)}=A_{\nu}^{(3)} B_{i}^{(1)}-B_{i}^{(1)} A_{\nu}^{(2)}=0, \\
& B_{i}^{(1)} B_{j}^{(3)}-B_{j}^{(1)} B_{i}^{(3)}=B_{i}^{(2)} B_{j}^{(1)}-B_{j}^{(2)} B_{i}^{(1)}=B_{i}^{(3)} B_{j}^{(2)}-B_{j}^{(3)} B_{i}^{(2)}=0, \\
& B_{i}^{(1)} B_{j}^{(1) \dagger}-B_{j}^{(2) \dagger} B_{i}^{(2)}=B_{i}^{(2)} B_{j}^{(2) \dagger}-B_{j}^{(3) \dagger} B_{i}^{(3)}=B_{i}^{(3)} B_{j}^{(3) \dagger}-B_{j}^{(1) \dagger} B_{i}^{(1)}=0 .
\end{aligned}
$$

There are two types of solutions to these equations. The first type has generally nonvanishing v.e.v. of $B_{i}$ and corresponds to the Higgs branch of super Yang-Mills theory. In this case, $A_{\mu}^{(\alpha)}$ must be independent of the block index $(\alpha)$ and has the form

$$
A_{\mu}^{(c l)}=\left(\begin{array}{ccc}
X_{\mu} & & \\
& X_{\mu} & \\
& & X_{\mu}
\end{array}\right),
$$

where $X_{\mu}$ are $N \times N$ diagonal matrices. The equation (4.2) can be satisfied if $B_{i}^{(\alpha)}$ is a diagonal matrix. To satisfy the last two equations (4.3) and (4.4), we further assume that $B_{i}^{(\alpha)}$ is independent of the block index $(\alpha)$ and has the form

$$
B_{i}^{(c l)}=\left(\begin{array}{ccc}
0 & 0 & B_{i}^{(2)(c l)} \\
B_{i}^{(3)(c l)} & 0 & 0 \\
0 & B_{i}^{(1)(c l)} & 0
\end{array}\right)=\left(\begin{array}{ccc}
0 & 0 & Z_{i} \\
Z_{i} & 0 & 0 \\
0 & Z_{i} & 0
\end{array}\right)
$$

where $Z_{i}$ is an $N \times N$ diagonal matrix. More generally, phases $\theta^{\alpha}$ of $B_{i}^{(\alpha)(c l)}$ can be put differently as $B_{i}^{(\alpha)(c l)}=e^{i \theta^{\alpha}} Z_{i}$ (where $\theta^{\alpha}$ is an $N \times N$ diagonal matrix). However, since they transform under $U(1)^{3 N}$ subgroup of the $U(N)^{3}$ symmetry as

$$
\theta_{\alpha} \rightarrow \theta_{\alpha}+\left(\phi_{\alpha+1}-\phi_{\alpha-1}\right)
$$

their relative phases can be gauged away. On the other hand, since the $\operatorname{sum} \sum_{i=1}^{3} \theta_{i}$ is invariant under these rotation, the common phase of $Z_{i}$ cannot be gauged away generally. We can only rotate the common phase of $Z_{i}$ by $2 \pi / 3$, which corresponds to changing the basis of $3 \times 3$ matrices. Writing the above solution (4.5) and (4.6) in terms of $U, V$ expansion, we have

$$
A_{\mu}^{(c l)}=X_{\mu} \otimes \mathbf{1}_{3 \times 3}, \quad B_{i}^{(c l)}=Z_{i} \otimes V .
$$


The second type of solution forms a singlet under $\mathbf{Z}_{3}$ rotation in $\mathbf{C}^{3}$ plane, and $B_{i}$ has vanishing vev. In this case, $A_{\mu}^{(c l)}$ can be an arbitrary diagonal matrix

$$
A_{\mu}^{(c l)}=\left(\begin{array}{ccc}
X_{\mu}^{(1)} & & \\
& X_{\mu}^{(2)} & \\
& & X_{\mu}^{(3)}
\end{array}\right)=\sum_{a=0}^{2} X_{\mu}^{a} \otimes U^{a} .
$$

This type of solutions corresponds to the Coulomb branch in the super Yang-Mills. These solutions are restricted on the orbifold fixed point and cannot move into $\mathbf{C}^{3}$ direction. This branch is connected with the Higgs branch solutions at $Z_{i}=0$ and $X_{\mu}^{(1)}=X_{\mu}^{(2)}=X_{\mu}^{(3)}$. When the Higgs branch solutions come to the orbifold fixed point, three images with the same four-dimensional coordinates are liberated, and become possible to move into $d=4$ direction independently from each other .

We then consider solutions with non-vanishing commutators $\left[A_{I}, A_{J}\right] \neq 0$. The first type (4.8) with the following commutators satisfies the equations of motion $\left[A^{I},\left[A_{I}, A_{J}\right]\right]=0$ :

$$
\left[X_{\mu}, X_{\nu}\right]=i C_{\mu \nu} \mathbf{1}_{N \times N},\left[Z_{i}, Z_{j}^{\dagger}\right]=D_{i j} \mathbf{1}_{N \times N},\left[Z_{i}, Z_{j}\right]=E_{i j} \mathbf{1}_{N \times N},\left[X_{\mu}, Z_{i}\right]=F_{\mu, i} \mathbf{1}_{N \times N},
$$

where $C_{\mu \nu}, D_{i j}, E_{i j}$ and $F_{\mu, i}$ are c-number coefficients. If $E_{i j}=F_{\mu, i}=0$, this solution becomes half BPS-saturated and preserves half of the supersymmetries. This solution corresponds to an ordinary D-brane.

The second type (4.9) is a classical solution if the commutator is proportional to a unit matrix

$$
\left[X_{\mu}^{(\alpha)}, X_{\nu}^{(\alpha)}\right]=i C_{\mu \nu}^{(\alpha)} \mathbf{1}_{N \times N}
$$

This solution is also half BPS-saturated if the coefficient $C_{\mu \nu}^{(\alpha)}$ is independent of the block index $(\alpha)$. An example is

$$
X_{0}^{(\alpha)}=\hat{p}, X_{1}^{(\alpha)}=\hat{q}, X_{2}^{(\alpha)}=x_{2}^{(\alpha)} \mathbf{1}_{N \times N}, X_{3}^{(\alpha)}=x_{3}^{(\alpha)} \mathbf{1}_{N \times N}
$$

where $[\hat{p}, \hat{q}]=-i$. It describes three fractional D-strings whose $x_{2}$ and $x_{3}$ coordinates can be different. They are constrained in the $z_{i}=0$ plane.

\subsection{Noncommutative Orbifold Field Theory}

Expanding our orbifold matrix model around the classical solutions (4.10), we can obtain noncommutative field theory in the orbifold background. Here we set $Z_{i}=0$, for simplicity. 
We write the classical solution $X_{\mu}$ as $\hat{x}_{\mu}$ to emphasise the noncommutativity of coordinates. Matrices are expanded around this noncommutative background as

$$
A_{\mu}=\left(\begin{array}{ccc}
\hat{x}_{\mu}+\tilde{A}_{\mu}^{(1)} & & \\
& \hat{x}_{\mu}+\tilde{A}_{\mu}^{(2)} & \\
& & \hat{x}_{\mu}+\tilde{A}_{\mu}^{(3)}
\end{array}\right) .
$$

All other matrices do not have classical background and are considered as fluctuating fields. All the fluctuating fields are written in terms of noncommutative plane waves in four dimensions as

$$
\tilde{A}_{\mu}^{a}=\sum_{k} \tilde{A}_{\mu}^{a}(k) \exp \left(i k_{\mu} \hat{x}_{\mu}\right) .
$$

By inserting these expansions and adopting the same technique to derive noncommutative field theory from IIB matrix model [12], the orbifold matrix model becomes noncommutative orbifold field theory with $\mathcal{N}=1$ supersymmetry in $d=4$. 《

If the background is replaced by a tensor product $\hat{x}_{\mu} \otimes \mathbf{1}_{n \times n}$, the resulting noncommutative field theory acquires $U(n)^{3}$ gauge symmetry. This model contains $d=4$ vector multiplet $\left(A_{\mu}^{a}\right.$ and $\left.\psi_{0}^{a}\right)$ in an adjoint representation of $U(n)^{3}$ and three chiral multiplets $\left(B_{i}^{a}\right.$ and $\left.\psi_{i}^{a}\right)$ in bi-fundamental representations. It is a chiral four dimensional theory. We may consider the number of chiral multiplets, three, as the number of generations (species) [14]. The global symmetry $U(3)$, which is a subgroup of $S O(6)$ part in $S O(9,1)$, becomes the horizontal symmetry among generations. Phenomenological analysis of this model will be left for future work (see also [13]).

We then consider classical solutions (4.12). In this case, if the positions of three fractional D-strings are largely separated, chiral multiplets $B_{i}$ and $\psi_{i}$ acquire large masses proportional to the distances, and only the vector multiplet survives in low energy. The low energy fields contain the same field contents as the $d=4$ super Yang-Mills gauge theory. Expanding around the D-string solution (4.12), the effective theory becomes $d=2$ noncommutative gauge theory obtained from the reduced matrix model of $d=4$ SYM theory. This is

\footnotetext{
${ }^{2}$ In these cases, $\hat{x}_{\mu}$ always enters in the action in a form of commutators. But if the background is also extended to the $\mathbf{C}^{3}$ direction and $B_{i}$ is expanded around noncommutative background $\hat{z}_{i}$, the same technique cannot be applied to obtain noncommutative field theory, because we are faced with a problem that the operator $\hat{z}_{i}$ enters into the action in a different form from commutators. Typically it is like $\left(\hat{z}_{i} \tilde{A}_{\mu}^{a}-\omega^{a} \tilde{A}_{\mu}^{a} \hat{z}_{i}\right)$ where the fluctuating fields are expanded in terms of the $U, V$ expansion. Hence, if we expand the fluctuating fields similarly, we cannot obtain an ordinary kinetic term. But by deforming the expansion basis of matrices from the ordinary noncommutative plane waves, we may be able to obtain well behaved field theory. A simple example of deformed plane wave is $M=\exp \left(i w \hat{a}^{\dagger}\right) q^{a^{\dagger} a} \exp (i \bar{w} \hat{a})$. This satisfies $a M-q M a=i w M$ where $\left[a, a^{\dagger}\right]=1$. Deformation of plane waves will be related to the resolution of orbifold singularity by the introduction of noncommutativity. Detailed analysis is now under investigation.
} 
consistent at least qualitatively with the fact that fractional branes have charges of RR fields in twisted sector, which propagate in $d=4$ subspace of $d=10$.

\subsection{Effective Action and Interaction between Diagonal Blocks}

We now calculate the one-loop effective action of orbifold matrix model between two diagonal blocks by the background field method. To fix gauge invariance in the perturbative calculation, we add the gauge fixing term

$$
S_{\mathrm{gf}}=-\frac{1}{g^{2}} \operatorname{Tr}\left(\frac{1}{2}\left[A_{I}^{c l}, \tilde{A}^{I}\right]^{2}+\left[A_{I}^{c l}, b\right]\left[A^{I, c l}, c\right]\right),
$$

where $b$ and $c$ are ghost fields and $A_{I}^{c l}$ includes background configurations of bosonic matrices. When we expand

$$
A_{\mu}=A_{\mu}^{c l}+\tilde{A}_{\mu}, B_{i}=B_{i}^{c l}+\tilde{B}_{i}
$$

the quadratic part of the quantum fluctuation in the bosonic action becomes

$$
\begin{aligned}
\tilde{S}_{b, 2}= & -\frac{1}{2 g^{2}} \operatorname{Tr}\left(\left[A_{I}^{c l}, \tilde{A}_{J}\right]\left[A^{I, c l}, \tilde{A}^{J}\right]+2\left[A_{I}^{c l}, A_{J}^{c l}\right]\left[\tilde{A}^{I}, \tilde{A}^{J}\right]\right) \\
= & -\frac{1}{2 g^{2}} \operatorname{Tr}\left(\left[A_{\mu}^{c l}, \tilde{A}_{\nu}\right]^{2}+\left[A_{\mu}^{c l}, \tilde{B}_{i}\right]\left[A^{\mu, c l}, \tilde{B}_{i}^{\dagger}\right]+\left[B_{i}^{c l}, \tilde{A}_{\mu}\right]\left[B_{i}^{c l \dagger}, \tilde{A}^{\mu}\right]\right. \\
& +\frac{1}{2}\left(\left[B_{i}^{c l}, \tilde{B}_{j}\right]\left[B_{i}^{c l \dagger}, \tilde{B}_{j}^{\dagger}\right]+\left[B_{i}^{c l}, \tilde{B}_{j}^{\dagger}\right]\left[B_{i}^{c l \dagger}, \tilde{B}_{j}\right]\right) \\
& +2\left(\left[A_{\mu}^{c l}, A_{\nu}^{c l}\right]\left[\tilde{A}^{\mu}, \tilde{A}^{\nu}\right]+\left[A_{\mu}^{c l}, B_{i}^{c l}\right]\left[\tilde{A}^{\mu}, \tilde{B}_{i}^{\dagger}\right]+\left[A_{\mu}^{c l}, B_{i}^{c l \dagger}\right]\left[\tilde{A}^{\mu}, \tilde{B}_{i}\right]\right) \\
& +\frac{1}{2}\left(\left[B_{i}^{c l}, B_{j}^{c l}\right]\left[\tilde{B}_{i}^{\dagger}, \tilde{B}_{j}^{\dagger}\right]+\left[B_{i}^{c l \dagger}, Z_{j}^{c l \dagger}\right]\left[\tilde{B}_{i}, \tilde{B}_{j}\right]\right) \\
& \left.+\left[B_{i}^{c l}, B_{j}^{c l \dagger}\right]\left[\tilde{B}_{i}^{\dagger}, \tilde{B}_{j}\right]\right) .
\end{aligned}
$$

We first consider the background of the first-type classical solution (4.8) with arbitrary $X_{\mu}$ and $Z_{i}$. Bosonic matrices are expanded as

$$
A_{\mu}=X_{\mu} \otimes \mathbf{1}_{3 \times 3}+\tilde{A}_{\mu}^{a} \otimes U^{a}, \quad B_{i}=Z_{i} \otimes V+\tilde{B}_{i}^{a} \otimes U^{a} V .
$$

Inserting these expansions into the action (4.17) and taking the trace over $3 \times 3$ matrices constructed by $U$ and $V$, we have

$$
\begin{aligned}
\tilde{S}_{b, 2}= & -\frac{3}{2 g^{2}} \operatorname{Tr}\left(\left[X_{\mu}, \tilde{A}_{\nu}^{a}\right]\left[X^{\mu}, \tilde{A}^{\nu,-a}\right]+\left[X_{\mu}, \tilde{B}_{i}^{a}\right]\left[X^{\mu}, \tilde{B}_{i}^{a \dagger}\right]\right. \\
& +\left(Z_{i} \tilde{A}_{\mu}^{a}-\omega^{a} \tilde{A}_{\mu}^{a} Z_{i}\right)\left(\omega^{-a} Z_{i}^{\dagger} \tilde{A}_{\mu}^{-a}-\tilde{A}_{\mu}^{-a} Z_{i}^{\dagger}\right) \\
& +\frac{1}{2}\left(\left(Z_{i} \tilde{B}_{j}^{a}-\omega^{a} \tilde{B}_{j}^{a} Z_{i}\right)\left(\omega^{-a} Z_{i}^{\dagger} \tilde{B}_{j}^{a \dagger}-\tilde{B}_{j}^{a \dagger} Z_{i}^{\dagger}\right)\right.
\end{aligned}
$$




$$
\begin{aligned}
& \left.+\left(Z_{i} \tilde{B}_{j}^{a \dagger}-\omega^{-a} \tilde{B}_{j}^{a \dagger} Z_{i}\right)\left(\omega^{a} Z_{i}^{\dagger} \tilde{B}_{j}^{a}-\tilde{B}_{j}^{a} Z_{i}^{\dagger}\right)\right) \\
+ & 2\left(\left[X_{\mu}, X_{\nu}\right]\left[A^{\tilde{\mu}, a}, \tilde{A}^{\nu,-a}\right]\right. \\
+ & {\left.\left[X_{\mu}, Z_{i}\right]\left(\omega^{-a} \tilde{A}^{\mu, a} \tilde{B}_{i}^{a \dagger}-\tilde{B}_{i}^{a \dagger} \tilde{A}^{\mu, a}\right)+\left[X_{\mu}, Z_{i}^{\dagger}\right]\left(\tilde{A}^{\mu, a} \tilde{B}_{i}^{-a}-\omega^{-a} \tilde{B}_{i}^{-a} \tilde{A}^{\mu, a}\right)\right) } \\
+ & \frac{1}{2}\left(\left[Z_{i}, Z_{j}\right] \omega^{a}\left(\tilde{B}_{i}^{a, \dagger} \tilde{B}_{j}^{-a, \dagger}-\tilde{B}_{j}^{a, \dagger} \tilde{B}_{i}^{-a, \dagger}\right)+\left[Z_{i}^{\dagger}, Z_{j}^{\dagger}\right] \omega^{a}\left(\tilde{B}_{i}^{a} \tilde{B}_{j}^{-a}-\tilde{B}_{j}^{a} \tilde{B}_{i}^{-a}\right)\right) \\
+ & {\left.\left[Z_{i}, Z_{j}^{\dagger}\right]\left(\tilde{B}_{i}^{a, \dagger} \tilde{B}_{j}^{a}-\tilde{B}_{j}^{a} \tilde{B}_{i}^{a, \dagger}\right)\right) . }
\end{aligned}
$$

Here $\operatorname{Tr}$ is a trace over $N \times N$ after taking the $3 \times 3$ part. Similarly the quadratic part in quantum fluctuation of the fermionic matrices becomes

$$
\begin{aligned}
\tilde{S}_{f, 2}= & -\frac{3}{2 g^{2}} \operatorname{Tr}\left(\sum_{i=0}^{3} \overline{\psi_{i}^{a}} \Gamma^{\mu}\left[X_{\mu}, \psi_{i}^{a}\right]+2 \sum_{i=1}^{3} \overline{\left(\psi_{i}^{a}\right)^{c}} \bar{\Gamma}^{(i)}\left(Z_{i}^{\dagger} \psi_{0}^{-a}-\omega^{a} \psi_{0}^{-a} Z_{i}^{\dagger}\right)\right. \\
& \left.+\sum_{i, j, k=1}^{3}\left|\epsilon_{i j k}\right| \overline{\left(\psi_{i}^{a}\right)^{c}} \Gamma^{(j)}\left(\omega^{-a} Z_{j} \psi_{k}^{-a}-\omega^{a} \psi_{k}^{-a} Z_{j}\right)\right)+ \text { h.c. }
\end{aligned}
$$

The last term survives due to the special property for the $\mathbf{Z}_{3}$ orbifold matrix model, $V^{3}=$ $\mathbf{1}_{3 \times 3}$.

We are interested in the induced interactions between backgrounds that are separated distantly and hence assume that $X_{\mu}$ and $Z_{i}$ are of $2 \times 2$ block form

$$
X_{\mu}=\left(\begin{array}{cc}
X_{\mu(1)} & \\
& X_{\mu(2)}
\end{array}\right), \quad Z_{i}=\left(\begin{array}{cc}
Z_{i(1)} & \\
& Z_{i(2)}
\end{array}\right) .
$$

The difference between the c-number parts of two blocks, $\left(\sum_{\mu}\left(x_{\mu(1)}-x_{\mu(2)}\right)^{2}+\sum_{i} \mid z_{i(1)}-\right.$ $\left.\left.z_{i(2)}\right|^{2}\right)^{1 / 2}$ is assumed to be much larger than the fundamental scale $g^{1 / 2}$ of the matrix model. In order to calculate interactions between these two blocks, we need to integrate over the fluctuations $\tilde{A}_{\mu}^{a}, \tilde{B}_{i}^{a}$ in the off-diagonal blocks;

$$
\tilde{A}_{\mu}^{a}=\left(\begin{array}{cc} 
& \tilde{A}_{\mu(12)}^{a} \\
\tilde{A}_{\mu(21)}^{a} &
\end{array}\right), \tilde{B}_{i}^{a}=\left(\begin{array}{cc} 
& \tilde{B}_{i(12)}^{a} \\
\tilde{B}_{i(21)}^{a} &
\end{array}\right) .
$$

In the quadratic actions (4.19), (4.20), $a=0$ sector is always decoupled from $a=1,2$ sector. This $a=0$ part is the same as the quadratic part of the IIB matrix model action with a gauge fixing term around the background $A_{\mu}=X_{\mu}, B_{i}=Z_{i}$. Hence integration over $a=0$ sector gives the same contribution as the block-block interactions in IIB matrix model (2.6), which can be interpreted as exchanges of massless particles in $d=10$ type IIB supergravity. The typical form is

$$
\frac{1}{\left(\left(x_{\mu(1)}-x_{\mu(2)}\right)^{2}+\left|z_{i(1)}-z_{i(2)}\right|^{2}\right)^{4}}\left(\operatorname{tr}\left(f_{\mu \nu(1)} f_{\nu \lambda(1)}\right) \operatorname{tr}\left(f_{\mu \sigma(2)} f_{\sigma \lambda(2)}\right)+\cdots\right) .
$$


The contributions from the other sectors $a=1,2$ can be evaluated similarly. First expand further the actions (4.19), 4.20) in terms of block fields (4.21), (4.22). The bosonic part becomes (we drop the coefficient $-3 / 2 g^{2}$ )

$$
\begin{aligned}
& \operatorname{Tr}\left(\left(X_{\mu(1)} \tilde{A}_{\nu(12)}^{a}-\tilde{A}_{\nu(12)}^{a} X_{\mu(2)}\right)\left(X_{(2)}^{\mu} \tilde{A}_{(21)}^{\nu,-a}-\tilde{A}_{(21)}^{\nu,-a} X_{(1)}^{\mu}\right)\right. \\
& +\left(X_{\mu(1)} \tilde{B}_{i(12)}^{a}-\tilde{B}_{i(12)}^{a} X_{\mu(2)}\right)\left(X_{(2)}^{\mu}\left(\tilde{B}_{i(12)}^{a}\right)^{\dagger}-\left(\tilde{B}_{i(12)}^{a}\right)^{\dagger} X_{(1)}^{\mu}\right) \\
& +\left(Z_{i(1)} \tilde{A}_{\mu(12)}^{a}-\omega^{a} \tilde{A}_{\mu(12)}^{a} Z_{i(2)}\right)\left(\omega^{-a} Z_{i(2)}^{\dagger} \tilde{A}_{\mu(21)}^{-a}-\tilde{A}_{\mu(21)}^{-a} Z_{i(1)}^{\dagger}\right) \\
& +\frac{1}{2}\left(\left(Z_{i(1)} \tilde{B}_{j(12)}^{a}-\omega^{a} \tilde{B}_{j(12)}^{a} Z_{i(2)}\right)\left(\omega^{-a} Z_{i(2)}^{\dagger}\left(\tilde{B}_{j(12)}^{a}\right)^{\dagger}-\left(\tilde{B}_{j(12)}^{a}\right)^{\dagger} Z_{i(1)}^{\dagger}\right)\right. \\
& \left.+\left(Z_{i(1)}\left(\tilde{B}_{j(21)}^{a}\right)^{\dagger}-\omega^{-a}\left(\tilde{B}_{j(21)}^{a}\right)^{\dagger} Z_{i(2)}\right)\left(\omega^{a} Z_{i(2)}^{\dagger} \tilde{B}_{j(21)}^{a}-\tilde{B}_{j(21)}^{a} Z_{i(1)}^{\dagger}\right)\right) \\
& +2\left(\left[X_{\mu(1)}, X_{\nu(1)}\right]\left(\tilde{A}_{(12)}^{\mu, a} \tilde{A}_{(21)}^{\nu,-a}-\tilde{A}_{(12)}^{\nu,-a} \tilde{A}_{(21)}^{\mu, a}\right)\right. \\
& +\left[X_{\mu(1)}, Z_{i(1)}\right]\left(\omega^{-a} \tilde{A}_{(12)}^{\mu, a}\left(\tilde{B}_{i(12)}^{a}\right)^{\dagger}-\left(\tilde{B}_{i(21)}^{a}\right)^{\dagger} \tilde{A}_{(21)}^{\mu, a}\right) \\
& \left.+\left[X_{\mu(1)}, Z_{i(1)}^{\dagger}\right]\left(\tilde{A}_{(12)}^{\mu, a} \tilde{B}_{i(21)}^{-a}-\omega^{-a} \tilde{B}_{i(12)}^{-a} \tilde{A}_{(21)}^{\mu, a}\right)\right) \\
& +\frac{1}{2}\left(\left[Z_{i(1)}, Z_{j(1)}\right] \omega^{a}\left(\left(\tilde{B}_{i(21)}^{a}\right)^{\dagger}\left(\tilde{B}_{j(12)}^{-a}\right)^{\dagger}-\left(\tilde{B}_{j(21)}^{a}\right)^{\dagger}\left(\tilde{B}_{i(12)}^{-a}\right)^{\dagger}\right)\right. \\
& \left.+\left[Z_{i(1)}^{\dagger}, Z_{j(1)}^{\dagger}\right] \omega^{a}\left(\tilde{B}_{i(21)}^{a} \tilde{B}_{j(12)}^{-a}-\tilde{B}_{j(21)}^{a} \tilde{B}_{i(12)}^{-a}\right)\right) \\
& +\left[Z_{i(1)}, Z_{j(1)}^{\dagger}\right]\left(\left(\tilde{B}_{i(21)}^{a}\right)^{\dagger} \tilde{B}_{j(21)}^{a}-\tilde{B}_{j(12)}^{a}\left(\tilde{B}_{i(12)}^{a}\right)^{\dagger}\right) \\
& +((1) \leftrightarrow(2),(12) \leftrightarrow(21))) .
\end{aligned}
$$

The fermionic part becomes

$$
\begin{aligned}
& \operatorname{Tr}\left(\sum_{i=0}^{3} \overline{\psi_{i(21)}^{a}} \Gamma^{\mu}\left(X_{\mu(2)} \psi_{i(21)}^{a}-\psi_{i(21)}^{a} X_{\mu(1)}\right)\right. \\
& +2 \sum_{i=1}^{3} \overline{\left(\psi_{i(12)}^{a}\right)^{c}} \Gamma^{(i)}\left(Z_{i(2)}^{\dagger} \psi_{0(21)}^{-a}-\omega^{a} \psi_{0(21)}^{-a} Z_{i(1)}^{\dagger}\right) \\
& \left.+\sum_{i, j, k=1}^{3}\left|\epsilon_{i j k}\right| \overline{\left(\psi_{i(12)}^{a}\right)^{c}} \Gamma^{(j)}\left(\omega^{-a} Z_{j(2)} \psi_{k(21)}^{-a}-\omega^{a} \psi_{k(21)}^{-a} Z_{j(1)}\right)\right) \\
& +((1) \leftrightarrow(2),(12) \leftrightarrow(21))+\text { h.c. }
\end{aligned}
$$

Then we can classify the fluctuating fields into three classes:

1. Fields with $a=0$,

2. $\tilde{A}_{\mu(12)}^{1}\left(=\left(\tilde{A}_{\mu(21)}^{2}\right)^{\dagger}\right), \tilde{B}_{i(12)}^{1}, \tilde{B}_{i(21)}^{2}, \tilde{\psi}_{i(12)}^{1}, \tilde{\psi}_{i(21)}^{2}$,

3. $\tilde{A}_{\mu(12)}^{2}\left(=\left(\tilde{A}_{\mu(21)}^{1}\right)^{\dagger}\right), \tilde{B}_{i(12)}^{2}, \tilde{B}_{i(21)}^{1}, \tilde{\psi}_{i(12)}^{2}, \tilde{\psi}_{i(21)}^{1}$. 
The quadratic actions are decoupled into three parts, each of which contains only the fields in each class. Furthermore the quadratic action for each class becomes the same as the $a=0$ case (class 1 ) when we absorb the $\mathbf{Z}_{3}$ phase $\omega^{a}$ into $Z_{i(2)}$ and redefine fields as

$$
\omega Z_{i(2)} \rightarrow Z_{i(2)}, \quad \omega \tilde{B}_{i(12)}^{1} \rightarrow \tilde{B}_{i(12)}^{1}, \quad \omega \tilde{\psi}_{i(12)}^{1} \rightarrow \tilde{\psi}_{i(12)}^{1}(i=1,2,3)
$$

for class 2 fields, and

$$
\omega^{2} Z_{i(2)} \rightarrow Z_{i(2)}, \quad \omega^{2} \tilde{B}_{i(12)}^{2} \rightarrow \tilde{B}_{i(12)}^{2}, \omega^{2} \tilde{\psi}_{i(12)}^{2} \rightarrow \tilde{\psi}_{i(12)}^{2}(i=1,2,3)
$$

for class 3 fields. Therefore, if the background is in the Higgs branch (4.18), we obtain the same block-block interactions as in IIB matrix model, except for the replacement of the typical interaction form (4.23) by

$$
\sum_{a=0}^{2} \frac{1}{\left(\left(x_{\mu(1)}-x_{\mu(2)}\right)^{2}+\left|z_{i(1)}-\omega^{a} z_{i(2)}\right|^{2}\right)^{4}}\left(\operatorname{tr}\left(f_{\mu \nu(1)} f_{\nu \lambda(1)}\right) \operatorname{tr}\left(f_{\mu \sigma(2)} f_{\sigma \lambda(2)}\right)+\cdots\right) .
$$

The $a \neq 0$ terms can be interpreted as interactions between the first block and the $\mathbf{Z}_{3}$ transformed mirror images of the second block. The tensor structure is the same as the IIB result (2.6) and each term can be interpreted as an interaction mediated by massless particles of type IIB supergravity in the untwisted sector in the orbifold background.

In the remainder of this subsection, we consider an effective action between Coulomb branch solutions or fractional branes in the same twisted sector. In matrix models, each twisted sector corresponds to each diagonal block matrices in (4.9). In string theory, fractional branes have charges of massless fields in the twisted sector, and the induced interaction is expected to have $d=4$ behaviour instead of $d=10$ behaviour. In the Coulomb branch, since the three fractional branes (4.12) can move freely into four-dimensional space-time, we can put them distantly from each other. Then the chiral multiplets including $B_{i}$ and $\psi_{i}$ become massive (mass is proportional to the distances) and are decoupled in the low energy. Only the vector multiplet of $A_{\mu}$ and $\psi_{0}$ survives. The low energy theory now becomes three decoupled reduced models of $d=4$ super Yang-Mills gauge theory. The effective interaction between fractional branes in the same twisted sector is therefore governed by this $\mathrm{d}=4$ reduced model. This behaviour is qualitatively consistent with the supergravity point of view. But in the perturbative calculation, the effective interaction is inversely proportional to fourth power of the distances (instead of quadratic) and inconsistent with the expected power of the supergravity calculation. It is not certain if we can overcome this difficulty nonperturbatively. 


\section{Recovery of IIB Matrix Model}

As we mentioned in the introduction, it is meaningful to make connection between IIB matrix model and the orbifold matrix model. If we can dynamically generate the orbifold matrix model from IIB matrix model, we could realize four-dimensional chiral fermion in IIB matrix model and we might also obtain some notion of universality class and background independence in matrix models. In this section we report their connection in the opposite direction that IIB matrix model can be obtained from the orbifold matrix model.

In the Higgs phase (4.8) with

$$
\begin{aligned}
A_{\mu}^{(c l)} & =X_{\mu} \otimes \mathbf{1}_{3 \times 3}=0, \\
B_{i}^{(c l)} & =Z_{i} \otimes V=z_{i} \mathbf{1}_{N \times N} \otimes V,
\end{aligned}
$$

where $z_{i}$ are complex numbers and $\left|z_{i}\right|^{2} \gg g, a \neq 0$ sector acquires a large mass and only $a=$ 0 sector survives. The resulting model becomes the IIB matrix model, and ten-dimensional Poincare invariance and $\mathcal{N}=2$ supersymmetry of IIB matrix model are recovered. This result is quite natural because in the large $z_{i}$ limit, the orbifold singularity will become invisible, and fields connecting different mirror sectors will have large mass proportional to the vev $\left|z_{i}\right|$.

We expand the matrices around the classical solution (5.1) as

$$
\begin{aligned}
A_{\mu} & =\tilde{A}_{\mu}^{a} \otimes U^{a}, \\
B_{i} & =z_{i} \mathbf{1}_{N \times N} \otimes V+\tilde{B}_{i}^{a} \otimes U^{a} V \\
\psi_{0} & =\psi_{0}^{a} \otimes U^{a} \\
\psi_{i} & =\psi_{i}^{a} \otimes U^{a} V,
\end{aligned}
$$

and insert them into the orbifold matrix model action (3.8). We then obtain the quadratic term,

$$
\begin{aligned}
\tilde{S}_{b, 2}= & -\frac{3}{4 g^{2}} \sum_{a=0, \pm 1}\left|\omega^{a}-1\right|^{2} \\
& \times \operatorname{Tr}\left[-2\left|z_{i}\right|^{2} \tilde{A}_{\mu}^{a} \tilde{A}_{\mu}^{-a}+\frac{1}{2}\left(-4\left|z_{i}\right|^{2} \tilde{B}_{j}^{a \dagger} \tilde{B}_{j}^{a}+2 z_{i} z_{j}^{*} \tilde{B}_{i}^{a \dagger} \tilde{B}_{j}^{a}-z_{i} z_{j} \tilde{B}_{i}^{a \dagger} \tilde{B}_{j}^{-a \dagger}-z_{i}^{*} z_{j}^{*} \tilde{B}_{i}^{a} \tilde{B}_{j}^{-a}\right)\right] \\
= & \frac{3}{2 g^{2}} \sum_{a=0, \pm 1}\left|\omega^{a}-1\right|^{2} z_{1}^{2} \operatorname{Tr}\left[\tilde{A}_{\mu}^{a} \tilde{A}_{\mu}^{-a}+\sum_{j=2}^{3} \tilde{B}_{j}^{a \dagger} \tilde{B}_{j}^{a}+\frac{1}{4}\left(\tilde{B}_{1}^{a}+\tilde{B}_{1}^{-a \dagger}\right)\left(\tilde{B}_{1}^{a \dagger}+\tilde{B}_{j}^{-a}\right)\right] .
\end{aligned}
$$

In the second line, we take $z_{1}$ real and $z_{2}=z_{3}=0$ by using the $U(3) \subset S O(6)$ invariance of 
the orbifold matrix model. Similarly, (3.10) gives the quadratic fermionic term

$$
\tilde{S}_{f, 2}=-\frac{3}{g^{2}} \sum_{a=0, \pm 1}\left(\omega^{-a}-1\right) z_{1}\left(\overline{\psi_{1}^{-a}} \Gamma^{(1)}\left(\psi_{0}^{a}\right)^{c}-\omega^{a} \overline{\left(\psi_{3}^{-a}\right)^{c}} \Gamma^{(1)} \psi_{2}^{a}\right)+\text { h.c. }
$$

All of the $a=0$ modes are massless, while $a \neq 0$ modes acquire large masses of the order of $z_{1}$ except one mode, $\tilde{B}_{1}^{a=1}-\left(\tilde{B}_{1}^{a=-1}\right)^{\dagger}$. This is a Nambu-Goldstone mode associated with the symmetry breaking $U(N)^{3} \rightarrow U(N)$ induced by the vev (5.1). Indeed, the vacuum transforms under $U(N)^{3}$ as

$$
\left[z_{1} \mathbf{1}_{N \times N} \otimes V, i \theta^{a} \otimes U^{a}\right]=i z_{1}\left(\omega^{-a}-1\right) \theta^{a} \otimes U^{a} V
$$

and $B_{1}^{a}=i z_{1}\left(\omega^{-a}-1\right) \theta^{a}$ satisfies the relation $\left(B_{1}^{a}\right)^{\dagger}=-B_{1}^{-a}$. As we will show in the remainder of this section, this Nambu-Goldstone mode disappears from the spectrum by the Higgs mechanism.

To discuss general structure of the action, we call the classical solution $Z$, massless modes in $a=0$ sector $L$, the above Nambu-Goldstone mode in $a \neq 0$ sector $G$, and massive modes in $a \neq 0$ sector $M$, in general. Then the triple and the quartic parts of the quantum fluctuation in the action have the following form, in general:

$$
\begin{aligned}
\tilde{S}_{b, 3} & =\left\{Z L L L, Z L G G, Z G G G, Z L L M, Z L G M, Z G G M, O\left(M^{2}\right)\right\} \\
\tilde{S}_{b, 4} & =\{L L L L, L L G G, L G G G, G G G G, O(M)\} \\
\tilde{S}_{f, 3} & =\{L L L, O(M)\} .
\end{aligned}
$$

The $L L L L$ terms in (5.7) and $L L L$ terms in (5.8) are exactly same as the IIB matrix model action. The $Z L L L$ terms in (5.6) vanish because $Z$ and $L$ commute. The $Z L L M$ terms in (5.6) vanish after taking a trace of $3 \times 3$ blocks because only $M$ is in $a \neq 0$ sector while $Z$ and $L$ are in $a=0$ sector.

Therefore, if we take unitary gauge, setting $G=0$, and integrate out the massive mode $M$, then we obtain the IIB matrix model action. If the $Z L L M$ terms were non-vanishing, these terms would induce new $L L L L$ terms, apart form the IIB matrix model action.

We can also see this Higgs mechanism in other gauges than the unitary gauge. In the large $z$ limit, the broken symmetry $U(N)^{3} / U(N)$ becomes the translational symmetry of the Nambu-Goldstone mode $G$. Therefore, the action, (5.6) and (5.7), should not depend on $G$, after we integrate out $M$. Indeed, $Z L G G, Z G G G$ terms in (5.6) vanish because

$$
\operatorname{Tr}\left[B_{1}, B_{1}^{\dagger}\right]^{2} \rightarrow 6 z_{1} \sum_{a=0, \pm 1}\left(1-\omega^{a}\right) \operatorname{Tr}\left(\tilde{B}_{1}^{a}+\tilde{B}_{1}^{-a \dagger}\right)\left[B_{1}, B_{1}^{\dagger}\right]^{-a}
$$


and this kind of terms must include $M$. Furthermore, after $M$ is integrated out, the ZLGM, ZGGM terms in (5.6) induce $L L G G, L G G G, G G G G$ terms, which cancel those terms in (5.7). This cancellation is easy to see in the $Z Z M M, Z G G M, G G G G$ terms:

$$
\begin{aligned}
S_{b} & \rightarrow \frac{3}{4 g^{2}}|1-\omega|^{2} \operatorname{Tr}\left[2 z_{1}^{2} M_{1}^{\dagger} M_{1}-\frac{1}{\sqrt{2}} z_{1}\left(M_{1} G^{2}+M_{1}^{\dagger} G^{\dagger 2}\right)+\frac{1}{4} G^{\dagger 2} G^{2}\right] \\
& =\frac{3}{4 g^{2}}|1-\omega|^{2} 2 \operatorname{Tr}\left(z_{1} M_{1}-\frac{1}{2 \sqrt{2}} G^{\dagger 2}\right)\left(z_{1} M_{1}^{\dagger}-\frac{1}{2 \sqrt{2}} G^{2}\right)
\end{aligned}
$$

where

$$
G=\frac{\tilde{B}_{1}^{1}-\tilde{B}_{1}^{-1 \dagger}}{\sqrt{2}}, M_{1}=\frac{\tilde{B}_{1}^{1}+\tilde{B}_{1}^{-1 \dagger}}{\sqrt{2}} .
$$

In this way, in the large $z_{i}$ limit, the Nambu-Goldstone mode disappears from the spectrum, only $a=0$ sector survive, and the IIB matrix model is recovered from the orbifold matrix model .

Finally in this section, we will shortly discuss the vacuum structure of the orbifold matrix model. We have shown that the orbifold matrix model is reduced to IIB matrix model in the Higgs branch with a large scalar vev. Is this vacuum dynamically stable? In field theory, this vacuum preserves half of supersymmetry and it is perturbatively stable. In the case of matrix model, we can discuss more about the stability of the vacuum. As studied in [10], eigenvalues of bosonic matrices in IIB matrix model tend to gather and no single eigenvalue can escape from the others. The dynamics of the eigenvalues in orbifold matrix model will also share the same property and then three mirror images of each eigenvalue will be bounded around the fixed point. If this is true, the vacuum with large vev in the Higgs branch is not realized dynamically and IIB matrix model is not recovered dynamically from the orbifold matrix model. Instead the nonperturbative vacuum of the orbifold matrix model will be such that the orbifold singularity cannot be neglected and have phenomenologically more interesting properties. It is more interesting if we can show that the orbifold matrix model can be dynamically generated from IIB matrix model. We want to come back to this problem.

\section{Conclusions and Discussions}

In this paper, we have studied matrix models in orbifold background, in particular, $\mathbf{C}^{3} / \mathbf{Z}_{3}$ orbifold with $\mathcal{N}=1$ four-dimensional supersymmetry. We find two types of classical solutions, the Higgs branch solution and the Coulomb branch solution. When the solutions have noncommutativity, they correspond to ordinary D-branes and fractional branes respectively. We then calculate the one-loop effective action around these solutions and show that in the 
former case we can correctly reproduce the supergravity result in the orbifold background. In the latter case, although we can obtain qualitatively consistent picture with the string theory, perturbative calculation does not reproduce the supergravity result. Finally we show that this model includes IIB matrix model in a special point of the perturbative moduli in a sense that the orbifold matrix model is reduced to IIB matrix model when the scalar fields $B_{i}$ have large expectation values in the Higgs branch.

There are several issues that are not studied in this paper. One of them is whether the orbifold matrix model is equivalent to the IIB matrix model. As we discussed in the introduction, various matrix models might be related to each other. Since IIB matrix model is obtained from the orbifold matrix model, we now want to show the reverse. That is to say, it is desirable if we can obtain the orbifold matrix model from IIB matrix model by some mechanism such as condensations of the fields. When we simply consider vacuum expectation values of scalar fields $B_{i}$ in IIB matrix model of size $3 N \times 3 N$, we cannot obtain four dimensional chiral theory. We have to violate parity and it is not yet known how we can twist the background to make the lower dimensional theory chiral. Perhaps we have to extend the size of IIB matrix model and consider parity violating condensation outside of the $3 N \times 3 N$ matrix. These are under investigations together with other mechanisms to generate four dimensional chiral fermions from IIB matrix model dynamically.

Another issue is resolution of the orbifold singularity by noncommutativity. As we briefly commented in the footnote 2, when we extend the space-time into the six dimensional transverse space, there is a singularity in the extended space. If we simply apply the same technique for deriving noncommutative field theories from matrix models as that used in IIB matrix model, we cannot obtain sensible field theory since the noncommutative coordinates enter into the action directly without commutators. But this problem will be overcome by adopting different type of noncommutative plane waves and this will be related to the resolution of singularity due to the noncommutativity of space. We discuss this problem in our future paper.

\section{Acknowledgements}

The work (H. Aoki and S. Iso) is supported in part by the Grant-in-Aid for Scientific Research from the Ministry of Education, Science and Culture of Japan. We thank N.Ishibashi for informing us of his thesis and J. Nishimura for discussions on dynamics of orbifold matrix models. 


\section{References}

[1] N. Ishibashi, H. Kawai, Y. Kitazawa and A. Tsuchiya, Nucl. Phys. B 498, 467 (1997) arXiv:hep-th/9612115.

[2] T. Eguchi and H. Kawai, Phys. Rev. Lett. 48, 1063 (1982).

[3] For review, see H. Aoki, S. Iso, H. Kawai, Y. Kitazawa, A. Tsuchiya and T. Tada, Prog. Theor. Phys. Suppl. 134, 47 (1999) arXiv:hep-th/9908038.

[4] Several mechanisms to generate chiral fermions are listed in the paper: A. M. Uranga, arXiv:hep-th/0201221.

[5] Field theories in this orbifold background was studied in the paper: S. Kachru and E. Silverstein, Phys. Rev. Lett. 80, 4855 (1998) arXiv:hep-th/9802183.

[6] There are several papers which studied on orbifolding matrix models. Itoyama and Tokura constructed USp matrix model by orbifolding IIB matrix model; H. Itoyama and A. Tokura, Prog. Theor. Phys. 99, 129 (1998) arXiv:hep-th/9708123; Phys. Rev. D 58, 026002 (1998) arXiv:hep-th/9801084.

$\mathrm{M}$ (atrix) theory on $\mathbf{S}^{1} / \mathbf{Z}_{2}$ was studied by N. Kim and S.J.Rey, Nucl.Phys. B504(1997) 189, hep-th/9701139; T. Banks and L. Motl, JHEP 9712 (1997) 003, hep-th/9703218; D. A. Lowe, Phys. Lett. B 403, 243 (1997) arXiv:hep-th/9704041. M(atrix) theory on $\mathbf{C}^{3} / \mathbf{Z}_{3}$ was studied by A. Miyake and A. Sugamoto, hep-th/0203223.

[7] Discrepancy in higher order terms between matrix theory calculation and supergravity result was reported in the paper; M. R. Douglas and H. Ooguri, Phys. Lett. B 425, 71 (1998) arXiv:hep-th/9710178.

[8] D. E. Diaconescu, M. R. Douglas and J. Gomis, JHEP 9802 (1998) 013 arXiv:hepth/9712230]. M. R. Douglas, B. R. Greene and D. R. Morrison, Nucl. Phys. B 506, 84 (1997) arXiv:hep-th/9704151. M. R. Douglas, JHEP 9707, 004 (1997) arXiv:hepth/9612126. M. R. Douglas, G. Moore, arXiv:hep-th/9603167.

[9] M. Fukuma, H. Kawai. Y. Kitazawa and A. Tsuchiya, Nucl Phys. B510 (1998), 158; hepth/9705128. 
[10] H. Aoki, S. Iso, H. Kawai, Y. Kitazawa and T. Tada, Prog. Theor. Phys. 99, 713 (1998) arXiv:hep-th/9802085].

[11] J. Nishimura and F. Sugino, arXiv:hep-th/0111102. J. Ambjorn, K. N. Anagnostopoulos, W. Bietenholz, F. Hofheinz and J. Nishimura, arXiv:hep-th/0104260. J. Nishimura and G. Vernizzi, JHEP 0004, 015 (2000) [arXiv:hep-th/0003223].

[12] H. Aoki, N. Ishibashi, S. Iso, H. Kawai, Y. Kitazawa and T. Tada, Nucl. Phys. B 565, 176 (2000) arXiv:hep-th/9908141. N. Ishibashi, S. Iso, H. Kawai and Y. Kitazawa, Nucl. Phys. B 573, 573 (2000) arXiv:hep-th/9910004. S. Iso, H. Kawai and Y. Kitazawa, Nucl. Phys. B 576, 375 (2000) arXiv:hep-th/0001027, M. Li, Nucl. Phys. B 499, 149 (1997) arXiv:hep-th/9612222.

[13] P. H. Frampton, arXiv:hep-th/0108011, G. Aldazabal, L. E. Ibanez, F. Quevedo and A. M. Uranga, JHEP 0008, 002 (2000) arXiv:hep-th/0005067.

[14] A realistic model with three generations from type I open string in $\mathbf{C}^{3} / \mathbf{Z}_{3}$ orbifold background was constructed by Ishibashi in his thesis. (unpublished, 1988) 